\title{
Imaging an Event Horizon: Mitigation of Scattering Toward Sagittarius A*
}

\section{Citation}

Fish, Vincent L., Michael D. Johnson, Ru-Sen Lu, Sheperd S. Doeleman, Katherine L. Bouman, Daniel Zoran, William T. Freeman, et al. 2014. "Imaging an Event Horizon: Mitigation of Scattering Toward Sagittarius A*." The Astrophysical Journal 795 (2) (October 22): 134. doi:10.1088/0004-637x/795/2/134. http://dx.doi.org/10.1088/0004-637X/795/2/134.

\section{Published Version}

doi:10.1088/0004-637X/795/2/134

\section{Permanent link}

http://nrs.harvard.edu/urn-3:HUL.InstRepos:27801848

\section{Terms of Use}

This article was downloaded from Harvard University's DASH repository, and is made available under the terms and conditions applicable to Other Posted Material, as set forth at http:// nrs.harvard.edu/urn-3:HUL.InstRepos:dash.current.terms-of-use\#LAA

\section{Share Your Story}

The Harvard community has made this article openly available.

Please share how this access benefits you. Submit a story.

\section{Accessibility}




\title{
IMAGING AN EVENT HORIZON: MITIGATION OF SCATTERING TOWARD SAGITTARIUS A*
}

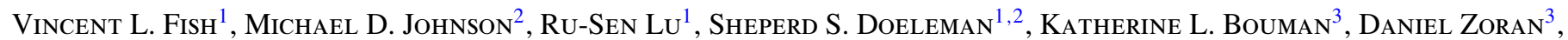 \\ William T. Freeman ${ }^{3}$, Dimitrios Psaltis ${ }^{4}$, Ramesh Narayan ${ }^{2}$, Victor Pankratius ${ }^{1}$, Avery E. Broderick ${ }^{5,6}$, \\ Carl R. GWinN ${ }^{7}$, and Laura E. Vertatschitsch ${ }^{2}$ \\ ${ }^{1}$ Massachusetts Institute of Technology, Haystack Observatory, Route 40, Westford, MA 01886, USA; vfish@ haystack.mit.edu \\ ${ }^{2}$ Harvard-Smithsonian Center for Astrophysics, 60 Garden Street, Cambridge, MA 02138, USA \\ ${ }^{3}$ Massachusetts Institute of Technology, Computer Science and Artificial Intelligence Laboratory, 32 Vassar Street, Cambridge, MA 02139, USA \\ ${ }^{4}$ Astronomy and Physics Departments, University of Arizona, 933 North Cherry Street, Tucson, AZ 85721, USA \\ ${ }^{5}$ Perimeter Institute for Theoretical Physics, 31 Caroline Street North, Waterloo, ON N2L 2Y5, Canada \\ ${ }^{6}$ Department of Physics and Astronomy, University of Waterloo, 200 University Avenue West, Waterloo, ON N2L 3G1, Canada \\ ${ }^{7}$ Department of Physics, University of California, Santa Barbara, CA 93106, USA \\ Received 2014 June 20; accepted 2014 September 15; published 2014 October 22
}

\begin{abstract}
The image of the emission surrounding the black hole in the center of the Milky Way is predicted to exhibit the imprint of general relativistic (GR) effects, including the existence of a shadow feature and a photon ring of diameter $\sim 50 \mu$ as. Structure on these scales can be resolved by millimeter-wavelength very long baseline interferometry. However, strong-field GR features of interest will be blurred at $\lambda \geqslant 1.3 \mathrm{~mm}$ due to scattering by interstellar electrons. The scattering properties are well understood over most of the relevant range of baseline lengths, suggesting that the scattering may be (mostly) invertible. We simulate observations of a model image of Sgr A* and demonstrate that the effects of scattering can indeed be mitigated by correcting the visibilities before reconstructing the image. This technique is also applicable to Sgr A* at longer wavelengths.
\end{abstract}

Key words: galaxies: individual (Sgr A*) - Galaxy: center - scattering - techniques: image processing techniques: interferometric

Online-only material: color figures

\section{INTRODUCTION}

The black hole in the center of the Milky Way, Sagittarius A* $\left(\operatorname{Sgr} \mathrm{A}^{*}\right)$, is the best candidate for spatially resolving a black hole image at horizon scales. With a mass of $\sim 4.3 \times 10^{6} M_{\odot}$ at a distance of $\sim 8.3 \mathrm{kpc}$ (Ghez et al. 2008; Gillessen et al. 2009a, 2009b), one Schwarzschild radius subtends $\sim 10 \mu$ as as viewed from the Earth. When illuminated by the hot material that surrounds it, general relativity (GR) predicts that a distant observer would see a bright photon ring enclosing a darker shadow region whose diameter is $\sim 50 \mu$ as (Bardeen 1973; Falcke et al. 2000). Though very small, this size scale is accessible to ground-based very long baseline interferometry (VLBI) at $\lambda \leqslant 1.3 \mathrm{~mm}$. Sgr $\mathrm{A}^{*}$ is one of the main targets of the Event Horizon Telescope (EHT), an international collaboration to spatially resolve and image the GR-dominated innermost accretion and outflow region around nearby supermassive black holes (Doeleman et al. 2009a).

There have been three motivations for the push toward shortwavelength VLBI of Sgr A*. First, the angular resolution of an interferometric baseline is $\lambda /|\mathbf{b}|$, where $|\mathbf{b}|$ is the projected baseline length perpendicular to the line of sight. The baseline length is limited by the size of the Earth for a ground-based array. At $1.3 \mathrm{~mm}$, the longest EHT baselines will provide an angular resolution of $\lesssim 25 \mu$ as. Increased angular resolution can be obtained by observing at shorter wavelengths. Second, the inferred intrinsic size of the emission from Sgr A* (37 $\mu$ as at $1.3 \mathrm{~mm}$; Doeleman et al. 2008) is larger at longer wavelengths (Doeleman et al. 2001; Bower et al. 2004; Shen et al. 2005), indicating that the observed emission is optically thick at longer wavelengths, obscuring the shadow near the black hole. Third and most problematically, interstellar scattering by free electrons blurs the image of Sgr A*, causing its apparent size to be proportional to $\lambda^{2}$, with the inferred diffractive scale of the scattering corresponding to baseline lengths of approximately $4500 \times 9300 \mathrm{~km}$ along the major and minor axes at $\lambda=1.3 \mathrm{~mm}$ and $1700 \times 3500 \mathrm{~km}$ at $\lambda=3.5 \mathrm{~mm}$ (Lo et al. 1998; Bower et al. 2004, 2006; Shen et al. 2005; Falcke et al. 2009; Lu et al. 2011; Akiyama et al. 2013). This effect dominates the size measurement at $\lambda \gtrsim 3.5 \mathrm{~mm}$ and is large enough to produce significant blurring even at $1.3 \mathrm{~mm}$ (where a point source would be scattered to be $\sim 22 \mu$ as in the long direction).

The sensitivity and baseline coverage of the EHT will increase dramatically over the next few years, especially with the inclusion of phased Atacama Large Millimeter/Submillimeter Array (ALMA) as a VLBI station (Fish et al. 2013), allowing EHT targets to be imaged. Simulated EHT data of the black hole in the nearby giant elliptical galaxy M87 have demonstrated that the array will be capable of imaging nearby black holes with sufficient fidelity to resolve the black hole shadows ( $\mathrm{Lu}$ et al. 2014). The size of the shadow in M87 is slightly smaller than in Sgr A*, but M87 is not significantly scatter-broadened. An important question that remains for Sgr A* is whether the fine details of the image will be irreversibly washed out by interstellar scattering (e.g., Broderick et al. 2011a; Yan et al. 2014). In this paper we demonstrate that the effects of interstellar scattering will be largely invertible in the case of $1.3 \mathrm{~mm} \mathrm{VLBI}$ of Sgr A*, allowing most features of the intrinsic structure to be recovered.

\section{SCATTER BROADENING}

\subsection{Theory}

Variations in the density of the tenuous interstellar plasma scatter radio waves, resulting in temporal and angular broadening of sources as well as scintillation in frequency and time (see, e.g., Rickett 1990). These variations impart a stochastic phase, $\phi(\mathbf{r})$, proportional to frequency and density variations, that changes across the scattering disk. Here, $\mathbf{r}$ is a transverse 
coordinate at the scattering screen. The variations are typically quantified by the phase structure function,

$$
D_{\phi}(\mathbf{r})=\left\langle\left[\phi\left(\mathbf{r}^{\prime}+\mathbf{r}\right)-\phi\left(\mathbf{r}^{\prime}\right)\right]^{2}\right\rangle .
$$

This structure function exhibits a power law $D_{\phi}(\mathbf{r}) \propto|\mathbf{r}|^{\alpha}$ over scales from $1000 \mathrm{~km}$ to over $1000 \mathrm{AU}$, suggesting a turbulent cascade (e.g., Armstrong et al. 1995). Strongly scattered sources often have a power-law index $\alpha$ close to 2 (the Kolmogorov index is $5 / 3$ ), which could arise from scattering by a medium consisting of discrete scatterers with abrupt boundaries or from scattering at wavelengths such that the phase coherence length $r_{0} \propto \lambda^{-2 / \alpha}$ on the scattering screen, defined such that $D_{\phi}(\mathbf{r}) \equiv 1$ for $|\mathbf{r}|=r_{0}$, is shorter than $r_{\text {in }}$, the dissipation scale of the turbulent cascade (Tatarskii 1971; Lambert \& Rickett 1999).

The dominant effects of scattering depend on the size of the source as well as the time and frequency resolution with which the source is observed (e.g., Narayan \& Goodman 1989; Goodman \& Narayan 1989). In the snapshot-image regime, observations of a very compact source with very high time and frequency resolution will detect stochastic variations in frequency and time due to diffractive scintillation. As the integration time or observed bandwidth is increased, the observing array effectively averages over multiple snapshot images. In this average-image regime, fast diffractive scintillation is suppressed, but visibilities fluctuate on significantly broader scales in frequency and time due to refractive scintillation. For still longer integration times or wider bandwidths, an interferometer averages over many realizations of the scattering screen. In this ensemble-average regime, the response of an interferometer to a point source is

$$
\tilde{I}(\mathbf{u})=\exp \left[-\frac{1}{2} D_{\phi}\left(\frac{\lambda \mathbf{u}}{1+M}\right)\right],
$$

where the tilde denotes quantities in the Fourier (visibility) domain, $\mathbf{u} \equiv(u, v)$ represents the projected baseline coordinates in units of the observing wavelength $\lambda$, and $M$ is the magnification factor of the scattering screen (the observer-scatterer distance divided by the source-scatterer distance).

Interstellar scattering is significant throughout the Galactic center region (e.g., van Langevelde et al. 1992). The consistency of angular broadening measurements with $\alpha \approx 2$ implies that ensemble-average scatter broadening is well approximated by a Gaussian. The scattering disk is anisotropic, as is typical for many lines of sight, which may indicate elongation of turbulent eddies along their local magnetic field (Goldreich \& Sridhar 1995).

The ideal ensemble-average scattering kernel (Equation (2)) is deterministic and purely real-valued. Departures of the scattering response from this ideal case can arise from diffractive or refractive effects. An extended source suppresses these types of scintillation noise (Gwinn et al. 1991, 1998). For Sgr A*, where the intrinsic $1.3 \mathrm{~mm}$ source size is much larger than the diffractive scale, the diffractive noise is negligible. Moreover, because the size of a scatter-broadened point source is smaller than that of the intrinsic structure at $1.3 \mathrm{~mm}$, the refractive noise is also partially quenched.

\subsection{Inversion of the Scattering Kernel}

By the van Cittert-Zernike theorem, the visibilities measured by interferometry are related to the Fourier transform of the sky image as

$$
\tilde{I}(\mathbf{u})=\int d^{2} \mathbf{x} I(\mathbf{x}) e^{-2 \pi i \mathbf{u} \cdot \mathbf{x}} .
$$

Convolution in the image domain is equivalent to multiplication in the visibility domain by the Fourier conjugate of the convolution kernel: $I(\mathbf{x}) * G(\mathbf{x}) \Leftrightarrow \tilde{I}(\mathbf{u}) \tilde{G}(\mathbf{u})$, where $*$ denotes convolution. The Fourier conjugate of the elliptical Gaussian scattering kernel $G(\mathbf{x})$ in the image domain is an elliptical Gaussian $\tilde{G}(\mathbf{u})$ in the visibility domain. Importantly, the scattering kernel is real $\left(\tilde{G}(\mathbf{u}) \in \mathbb{R}^{+}\right.$for all $\left.\mathbf{u}\right)$ and decreases monotonically in all directions. The net effect is that long-baseline amplitudes of the scattered image are lower than would be measured for the unscattered image, but visibility phases are unaffected.

Because the elliptical scattering kernel is strictly positive, its effects are invertible. Measured visibilities can be divided by $\tilde{G}(\mathbf{u})$ to recover estimates of the visibilities of the unscattered image. Of course, the loss in the signal-to-noise ratio, $\mathrm{S} / \mathrm{N} \equiv \tilde{I}(\mathbf{u}) / \tilde{N}(\mathbf{u})$, where $\tilde{N}(\mathbf{u})$ represents the noise of the measured visibility, cannot be recovered, since the interferometer senses the scattered image. This places a natural limit on the applicability of the inversion technique. At very large $\mathbf{u}, \mathrm{S} / \mathrm{N} \rightarrow 0$ and $\tilde{G}(\mathbf{u}) \rightarrow 0$, with the result that division by $\tilde{G}(\mathbf{u})$ amplifies the noise (although such data points would have very little weight in most image reconstruction algorithms due to their low $\mathrm{S} / \mathrm{N}$ ). This limit is not applicable to ground-based VLBI of Sgr A* at $1.3 \mathrm{~mm}$, where $\tilde{G}(\mathbf{u})>0.19$ over the entire range of $\mathbf{u}$ that will be covered by EHT baselines (Figure 1).

An alternate strategy is to first reconstruct the scattered image and then attempt deconvolution. To obtain the unscattered image, we must solve an inverse problem that deconvolves the known scattering $G(\mathbf{x})$ from the scattered image. This inverse problem, termed non-blind deconvolution, has been studied extensively in signal and image processing. Without noise the solution to the inverse problem is trivial: Fourier transform the image, divide by $\tilde{G}(\mathbf{u})$, and inverse transform back to the image domain. However, in practice, noise dominates the high spatial frequencies of a reconstructed image. Dividing by $\tilde{G}(\mathbf{u})$ strongly amplifies this noise, introducing potentially severe artifacts into the image. Many different non-blind deconvolution approaches exist that vary greatly in their speed and sophistication in order to address this problem (e.g., Krishnan \& Fergus 2009; Zoran \& Weiss 2011; Joshi et al. 2009), but the Wiener deconvolution filter is perhaps the simplest and most general deconvolution approach (Wiener 1949). The Wiener filter,

$$
\tilde{G}_{\mathrm{W}}^{-1}(\mathbf{u})=\frac{1}{\tilde{G}(\mathbf{u})}\left[\frac{|\tilde{G}(\mathbf{u})|^{2}}{|\tilde{G}(\mathbf{u})|^{2}+K}\right],
$$

can be defined in terms of a tunable parameter $K$ (Russ 2011). As $K \rightarrow 0, \tilde{G}_{\mathrm{W}}^{-1}(\mathbf{u}) \rightarrow \tilde{G}^{-1}(\mathbf{u})$, resulting in a sharper image but potentially unbounded amplification of high-frequency noise. In theory, the optimal value of $K$ is inversely proportional to the square of $\mathrm{S} / \mathrm{N}$, but the noise in the Fourier domain is neither constant nor known a priori. Indeed, the noises of the visibilities from which the image is reconstructed are typically unequal due to differing telescope sensitivities, and the image reconstruction process (which must necessarily fill in information from unmeasured Fourier components) introduces additional noise in the Fourier domain. In practice, $K$ is treated as a tunable parameter. ${ }^{8}$

\footnotetext{
8 When an image is reconstructed from corrected visibilities, the $\mathrm{S} / \mathrm{N}$ of each data point is retained, so highly amplified low-S/N data do not corrupt the reconstructed image. In contrast, when an image is reconstructed from the uncorrected visibilities and deconvolution is attempted in the image domain, the $\mathrm{S} / \mathrm{N}$ information associated with each Fourier component is not preserved.
} 

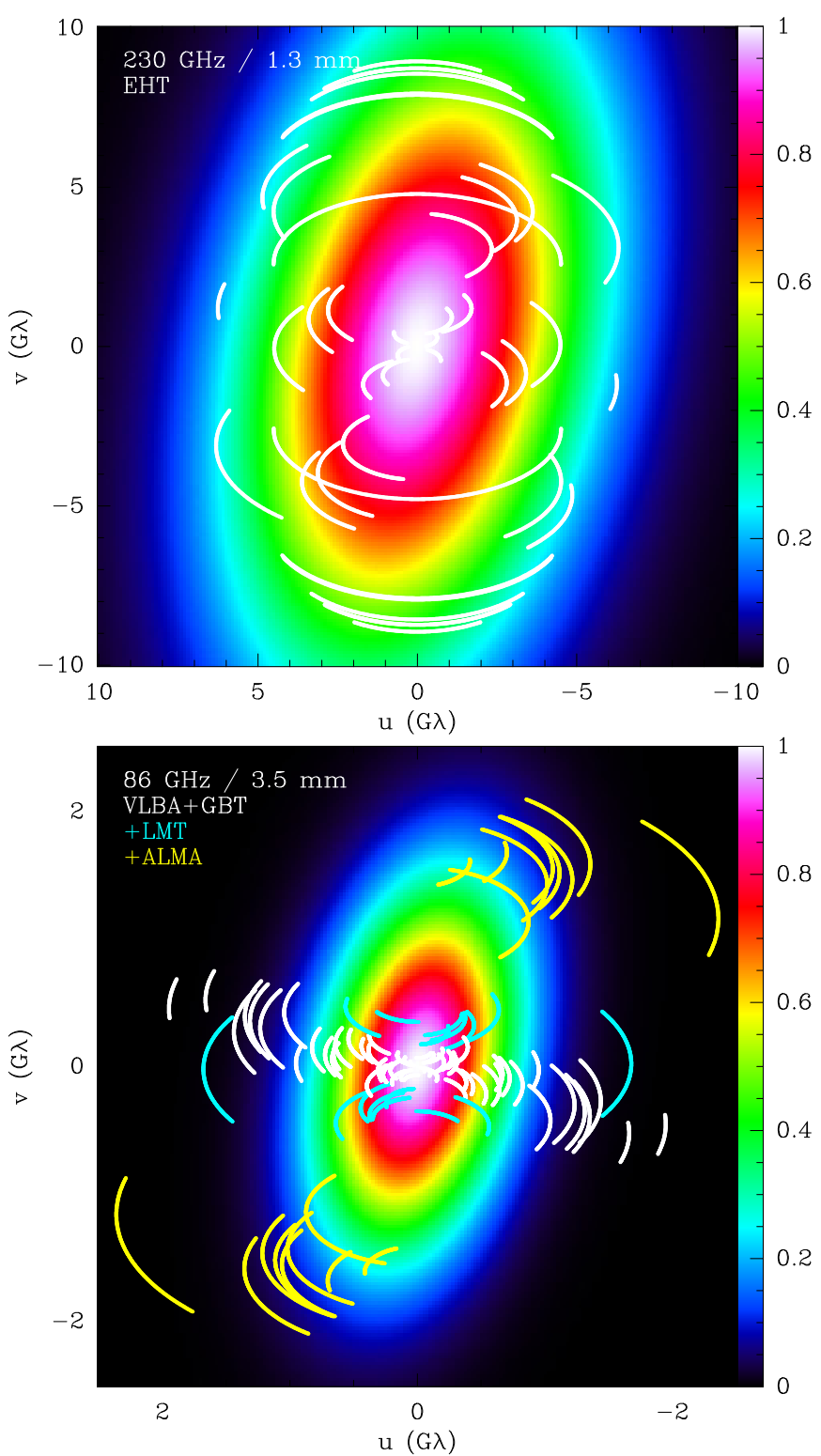

Figure 1. Plot of the elliptical Gaussian scattering kernel $\tilde{G}(\mathbf{u})$ in the $(u, v)$ plane at $1.3 \mathrm{~mm}$ (top) and $3.5 \mathrm{~mm}$ (bottom) with baseline tracks overplotted. White tracks show the $(u, v)$ coverage attainable with the EHT (including the LMT and ALMA) at $1.3 \mathrm{~mm}$ and the VLBA plus GBT at $3.5 \mathrm{~mm}$. The addition of the LMT (cyan) to the $3.5 \mathrm{~mm}$ observing array provides many baseline tracks that are not heavily scattered. Baselines from ALMA (yellow) to the LMT, GBT, and some VLBA stations may also have detectable flux.

(A color version of this figure is available in the online journal.)

\section{METHODS}

We demonstrate how scattering may be mitigated in practice by simulating observations of $\operatorname{Sgr} \mathrm{A} *$ at $1.3 \mathrm{~mm}$. We include the effects of scattering, generate synthetic data, correct the visibility amplitudes, reconstruct the image, and assess image fidelity relative to the model image.

\subsection{Data Simulation}

Our input image is a semi-analytic radiatively inefficient accretion flow model of Sgr $\mathrm{A}^{*}$ using the best-fit model parameters from Broderick et al. (2011a). This image was scattered using the parameters given by Bower et al. (2006). Simulated $1.3 \mathrm{~mm}$ EHT data were produced using the MIT Array
Performance Simulator. ${ }^{9}$ Telescopes in the simulated array included the Submillimeter Array and James Clerk Maxwell Telescope on Mauna Kea, the Arizona Radio Observatory Submillimeter Telescope, the Combined Array for Research in Millimeter-wave Astronomy, the Large Millimeter Telescope (LMT), ALMA, the Institut de Radioastronomie Millimétrique (IRAM) $30 \mathrm{~m}$ telescope on Pico Veleta, the IRAM Plateau de Bure Interferometer, and the South Pole Telescope. Further details of the simulated observing array can be found in Lu et al. (2014).

Our simulations include realistic thermal noise but neglect the effect of calibration errors on visibility amplitudes. It is difficult to estimate what the magnitude of calibration errors will be in the era when the EHT has enough sensitivity and baseline coverage to image Sgr $\mathrm{A}^{*}$. In contrast with the anticipated capabilities of the EHT in the next few years, previously published EHT observations of Sgr A* (Doeleman et al. 2008; Fish et al. 2011) are based on comparatively low$\mathrm{S} / \mathrm{N}$ data taken with an array consisting of only three VLBI sites. Accurate amplitude calibration has been challenging for a variety of reasons, such as the limited sensitivity of the array and the paucity of redundancy in the data. The EHT of the near future will almost certainly do better through a combination of higher sensitivity, enabled by much wider bandwidths; scheduling designed to improve a priori data calibration, itself enabled by higher sensitivity; calibration constraints provided by amplitude closure, which requires at least four VLBI stations; imaging algorithms which treat closure amplitudes as fundamental observables that are inherently robust against amplitude calibration errors; use of prior phase information to provide partial phase calibration of the array; and optimized data processing. Initial images of the quiescent millimeter-wavelength emission from $\mathrm{Sgr} \mathrm{A}^{*}$ are likely to be of lower fidelity than presented in this work for a number of reasons, including calibration errors, refractive phase noise on long baselines (Section 5.1), and rapid source variability (Section 5.2). Nevertheless, it is instructive to examine scattering mitigation on ideal simulated data in order to explore the possibilities and limitations of mitigation techniques.

\subsection{Image Reconstruction}

The simulated data were imaged using the BiSpectrum Maximum Entropy Method (BSMEM; Buscher 1994), as described in detail in Lu et al. (2014). Developed by the infrared and optical interferometry community, BSMEM differs from classical centimeter-wavelength interferometric imaging techniques in two key ways that are well suited to millimeter-wavelength VLBI imaging. First, variations in the tropospheric delay impose phase fluctuations that are too rapid to calibrate out using standard phase-referencing techniques. Since the atmospheric phase contributions are antenna-based, their contributions cancel along a closed loop of antennas (e.g., Jennison 1958; Rogers et al. 1974). BSMEM thus treats closure phases on triangles of stations, rather than visibility phases on baselines, as the fundamental phase observables for imaging. Second, the early EHT, like optical interferometers, will have sparse $(u, v)$ coverage. Deconvolution-based imaging techniques such as CLEAN (Högbom 1974) and Multi-Scale CLEAN (Cornwell 2008; Greisen et al. 2009) reconstruct an image by Fourier inverting the sampled visibilities to produce the so-called dirty map and then deconvolving the dirty beam

\footnotetext{
9 http://www.haystack.mit.edu/ast/arrays/maps/index.html
} 

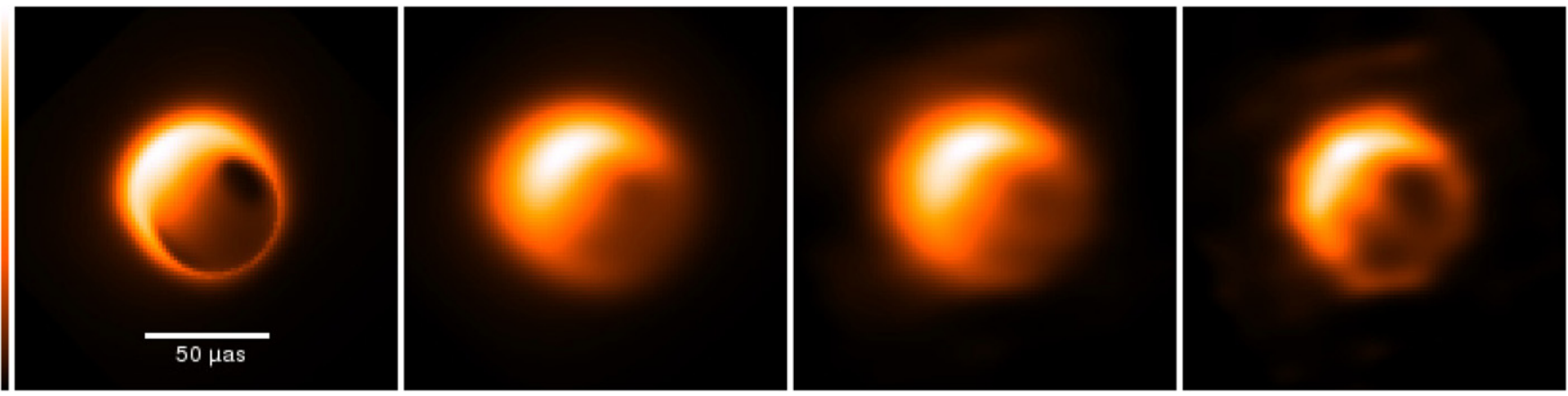

Figure 2. Imaging simulation of Sgr A* with the EHT. The model image (first panel) for a semi-analytic accretion flow around Sgr A* (Broderick et al. 2011a) is convolved with the known scattering kernel (second; MSE 0.087 and DSSIM 0.117 with first panel as the reference image). The BSMEM reconstruction of the scattered image (third; MSE 0.099, DSSIM 0.154) can be improved upon by dividing each synthetic visibility by the Fourier transform of the scattering kernel; the subsequent BSMEM reconstruction produces an image (fourth; MSE 0.024, DSSIM 0.076) much closer to the unscattered original. The image reconstruction using the corrected visibilities is able to clearly detect the shadow and photon ring associated with the black hole. A linear transfer function (far left) is used in each panel in this and subsequent figures.

(A color version of this figure is available in the online journal.)

(also known as the point-spread function) in the image domain. These techniques work well when imaging simple structures with arrays consisting of a large number of antennas (e.g., the Jansky Very Large Array). However, when the array consists of very few antennas, there are large unsampled areas in the $(u, v)$ plane, producing a dirty beam that has large sidelobes. In any case, the dirty beam is not positive-definite. Small errors during deconvolution can thus produce large artifacts in the image domain, severely limiting image fidelity. In contrast, forward imaging methods such as BSMEM avoid deconvolution by finding best-fit images that are directly consistent with the observables, using a priori knowledge and suitable regularizers. Unlike with CLEAN, images reconstructed with BSMEM are not normally convolved with a restoring beam, which in this case would significantly degrade the resolution of the reconstructed images.

\subsection{Image Fidelity Analysis}

We assessed image quality using both pixel-based and feature-based metrics. In the first category, the mean square error (MSE) quantifies the mean square pixel-by-pixel intensity difference between a truth image and a reconstructed image, normalized by the sum of the squares of the pixel intensities in the reference image. The MSE is equivalent to the metric used to assess image quality in the biennial interferometric imaging beauty contest (Baron et al. 2012, most recently). In the second category, motivated by human visual perception, the structural dissimilarity (DSSIM) index, derived from the structural similarity (SSIM) index (Wang et al. 2004; Łoza et al. 2009), quantifies differences in luminance, contrast, and structure between two images. Formal definitions of the MSE and DSSIM metrics are given in Lu et al. (2014). For both indices, lower values correspond to better image quality. Because absolute positional information is lost when reconstructing images using closure phase information, the images are cross-registered to the reference image at sub-pixel accuracy before calculating these metrics.

As an additional measure of artifacts introduced by Wiener deconvolution, we will also quote the ratio of the largest positive pixel to the absolute value of the largest negative pixel $(\mathrm{max} / \mathrm{min})$. This metric is not directly applicable to images reconstructed with BSMEM, which are constrained to be positive-definite.

\section{RESULTS}

We seek to answer three questions. First, does using corrected visibility amplitudes mitigate the effects of scatter broadening? Second, do corrected visibilities produce a higher-fidelity image than Wiener deconvolution of the scatter-broadened image? Third, are uncertainties in the scattering parameters at $1.3 \mathrm{~mm}$ small enough to permit scattering mitigation by these methods?

Correcting visibility amplitudes does indeed partially mitigate the effects of scatter broadening. The reconstructed image from the corrected visibilities is much sharper than would be recovered from the uncorrected visibilities (Figure 2), as confirmed by the MSE (0.099 uncorrected, 0.024 corrected) and DSSIM (0.154 uncorrected, 0.076 corrected) values. The image reconstructed from the corrected visibilities is able to recover the important physical features introduced by GR, including the dark shadow of the black hole and the associated bright photon ring at its edge (Broderick \& Loeb 2006; Dexter et al. 2009; Johannsen \& Psaltis 2010a, 2010b). Although these features are present in the reconstruction produced from the uncorrected visibilities, they are much less prominent, reflecting the fact that they are also less prominent in the scattered model image itself.

Correcting the visibilities before imaging fares better than reconstructing the scattered image and attempting deconvolution in the image domain (Figure 3). As the Wiener deconvolution parameter $K$ is lowered, features in the deconvolved image become sharper, but the strength of artifacts introduced by deconvolution become enhanced. A key distinction between these scattering mitigation methods is that the image directly reconstructed from corrected visibilities is positive-definite, while the deconvolved images have negative-pixel artifacts.

To determine whether uncertainties in the properties of the scattering are large enough to hamper mitigation, we considered three scattering kernels at $1.3 \mathrm{~mm}$ : a fiducial kernel from Bower et al. $\left(2006 ; 1.309 \times 0.64 \mathrm{mas} \mathrm{cm}^{-2}\right.$ at $78^{\circ}$ east of north), a small

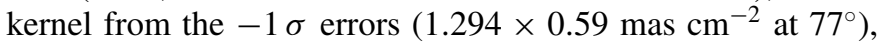
and a large kernel from the $+1 \sigma$ errors of Shen et al. (2005; $1.41 \times 0.75 \mathrm{mas} \mathrm{cm}^{-2}$ at $80^{\circ}$ ). The source image was convolved and Wiener deconvolved with different combinations of these kernels (Figure 4). In order to isolate and clearly illustrate the effects of mitigating scattering with incorrect parameters, convolution and deconvolution were applied to the model image itself rather than the BSMEM reconstruction thereof. Under-removing scattering results in a slightly more blurred 

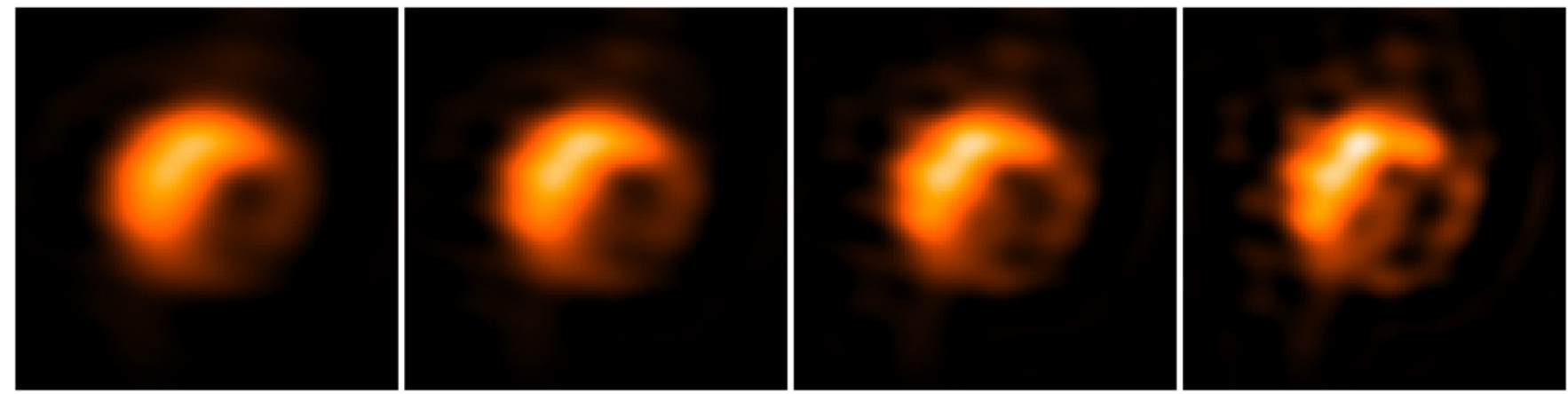

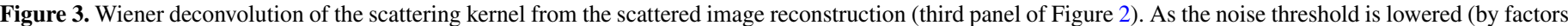

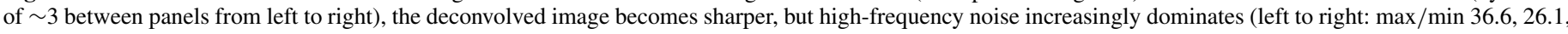

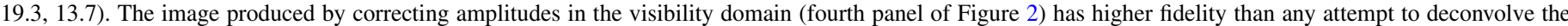

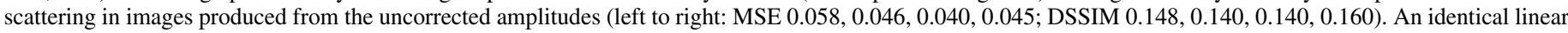
transfer function is used in all panels.

(A color version of this figure is available in the online journal.)
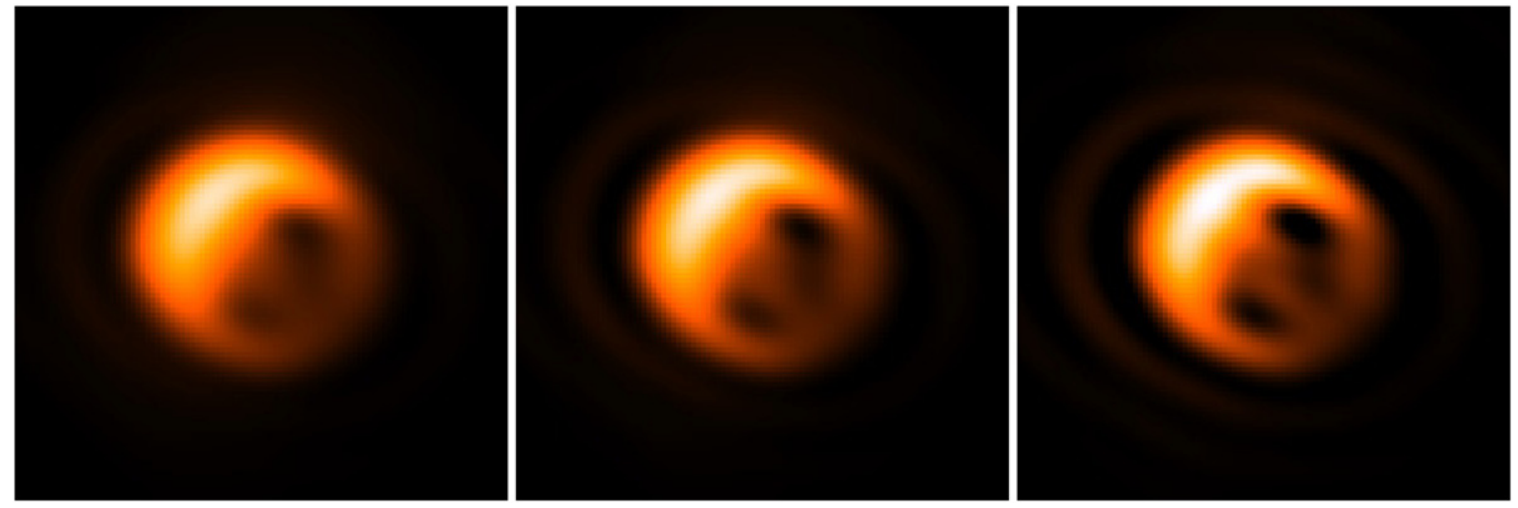

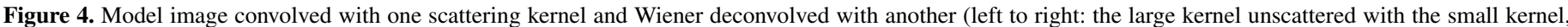

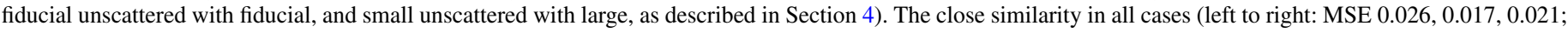

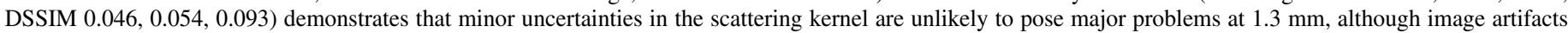
become significantly more prominent if the image is overcorrected with Wiener deconvolution (left to right: $\mathrm{max} / \mathrm{min} 533.6,304.7$, 18.4).

(A color version of this figure is available in the online journal.)

image than ideal, but the result is nevertheless a substantial improvement upon the scattered model image. Over-removing scattering results in a sharper image of $\mathrm{Sgr} \mathrm{A}^{*}$ at the expense of substantially larger image artifacts. Nevertheless, all cases significantly improve upon the resulting image as measured by both the MSE and DSSIM metrics.

\section{DISCUSSION}

\subsection{Validity of the Scattering Approximation}

We have demonstrated that the effects of idealized interstellar scattering of Sgr $\mathrm{A}^{*}$ at $1.3 \mathrm{~mm}$ can be partially mitigated by correcting visibilities before imaging. However, there are two potential ways in which real scattering may deviate from this approximation on long baselines: the phase structure function may deviate from an $\alpha \approx 2$ power law, and the scattering kernel may introduce refractive noise. We now estimate the influence of each of these uncertainties.

The phase structure function can be better characterized by continued observations at longer wavelengths and on nearby targets. As previously discussed, the scattering response must be an anisotropic Gaussian on short baselines. On longer baselines, the most plausible transition is to a Kolmogorov spectrum, which predicts that the image will be less scattered (and therefore that scattering mitigation will be less important) than would be predicted by the Gaussian regime. Because the scattering for $\operatorname{Sgr} \mathrm{A}^{*}$ at $1.3 \mathrm{~mm}$ may have $r_{0} \sim r_{\text {in }}$, improved understanding of the scattering will be vital for baselines $\gtrsim 3 \mathrm{G} \lambda$. The recent discovery of substructure in the scattered image of $\mathrm{Sgr} \mathrm{A}^{*}$ at $1.3 \mathrm{~cm}$ wavelength, which supports the Kolmogorov transition at wavelengths near a millimeter, provides one promising avenue to better characterize the scattering (Gwinn et al. 2014). Deeper studies of similarly scattered objects, such as the Galactic center magnetar SGR J1745-29, may also provide guidance (Bower et al. 2014). In any case, the amplitude portion of the scattering kernel $\tilde{G}(\mathbf{u})$ will be a predictable function of $\alpha, r_{0}$, and $r_{\text {in }}$ (Equation (3.1.1) of Goodman \& Narayan 1989). Fine adjustments of the scattering kernel, and thus the unscattered image, can be achieved through blind-deconvolution methods (Levin et al. 2009, 2011).

We can also estimate the level of refractive noise for Sgr A*. The $\lambda^{2}$ scaling of the scattering at longer wavelengths suggests that the inner scale of the turbulence is larger than the phase coherence length on the scattering screen. Taking $\alpha=5 / 3$ in the turbulent regime, the rms refractive noise $\sigma_{\mathrm{R}}(\mathbf{u}) \equiv\left\langle\sqrt{|\Delta \tilde{I}(\mathbf{u})|^{2}}\right\rangle$, normalized by the zero-baseline flux, for a point source is

$$
\sigma_{\mathrm{R}}(\mathbf{u}) \approx 0.37\left(\frac{r_{0}}{r_{\mathrm{F}}}\right)^{1 / 3}\left(\frac{|\mathbf{u}| \lambda}{(1+M) r_{0}}\right)^{-5 / 6}\left(\frac{r_{\text {in }}}{r_{0}}\right)^{1 / 6}
$$


(Equation (5.1.2) of Goodman \& Narayan 1989). At $1.3 \mathrm{~mm}$, the Fresnel scale is

$$
r_{\mathrm{F}}=\sqrt{\frac{d}{k} \frac{M}{(1+M)^{2}}} \approx 10^{5} \mathrm{~km}
$$

for $M \sim 1$ (Bower et al. 2014). The inner scale is most likely hundreds of kilometers (see Spangler \& Gwinn 1990), especially in light of recent measurements that disfavor a unique scattering environment (Bower et al. 2014). This replacement anticipates $\sigma_{\mathrm{R}}(\mathbf{u}) \approx 0.15$ on a $3 \mathrm{G} \lambda$ east-west baseline, for instance.

The refractive noise is correlated among different baselines, with a decorrelation length comparable to the diffractive scale (Goodman \& Narayan 1989). For a particular realization of the refractive noise, it will be correlated on all EHT baselines (Figure 1). Thus, by normalizing measurements by the zerobaseline flux, the noise on long baselines will be partially suppressed.

Refractive noise will additionally be suppressed by the finite (non-pointlike) extent of Sgr A*. Thus, even on long baselines $(\gtrsim 3 \mathrm{G} \lambda)$, the refractive phase "jitter" may be a $10^{\circ}$ or less with a characteristic timescale of variation of about one day (the refractive timescale). This phase would be constant over the duration of an observing night and across the entire observing bandwidth. The most sensitive evidence of these effects may be zero-mean variations in closure phase on timescales that are compatible with the refractive timescale (but see Section 5.2). Larger variations could indicate atypical properties of the turbulence that gives rise to the scattering along the line of sight to the Galactic center.

Refractive phase noise may introduce a small jitter in the apparent position of Sgr A*. This may introduce additional noise into measurements of the position wander of $\mathrm{Sgr} \mathrm{A}^{*}$ on interday timescales but will be constant on shorter timescales corresponding to orbital periods in the accretion flow. It will also be negligible when averaged over the longer timescales necessary for measuring the parallax and proper motion of Sgr A* (Reid et al. 2008; Broderick et al. 2011b).

Since refractive phase noise will have zero mean, a strategy to mitigate the effects of refractive phase noise is to observe Sgr A* for multiple nights. Phase quantities measured at identical points in the $(u, v)$ plane can be averaged across all observing nights to produce a single data set. As the particular realizations of refractive phase noises will be different from day to day, averaging many days of data together effectively produces a data set that is sensitive to the idealized (real) ensembleaverage image scattering regime. As the number of nights of data increases, the scattering kernel will asymptote to the real, invertible ensemble-average kernel.

\subsection{Connections to Variability}

Refractive noise will introduce small distortions into the observed image of Sgr A*. However, these distortions will be small compared to intrinsic changes in the emitting material around the black hole. Sgr A* exhibits variability and flaring activity across the spectrum (e.g., Eckart et al. 2006). At millimeter wavelengths, this is likely accompanied by structural changes or the appearance of hot spots in the accretion flow. GR magnetohydrodynamic simulations of the dynamic accretion flow (e.g., Noble et al. 2007; Mościbrodzka et al. 2009; Dexter et al. 2010) demonstrate that inhomogeneities can imprint large changes upon measured closure phases, as can nonthermal hot-spots (Broderick \& Loeb 2005; Doeleman et al. 2009b).
These variations will be evident on timescales of minutes. The innermost stable circular orbital period of Sgr A* is about half an hour if the black hole spin is close to zero and shorter still if the black hole is spinning rapidly, and the light-crossing time for a region that is one gravitational radius across is $G M c^{-3} \approx 20 \mathrm{~s}$.

Standard aperture-synthesis techniques rely on Earth rotation to change the projected baselines over the course of many hours, providing greater coverage in the $(u, v)$ plane and hence higher imaging fidelity. However, visibilities obtained at different times will correspond to different images. Naive application of the Earth-rotation aperture-synthesis technique may fail because there will not be a single image that is consistent with all measured visibilities in the $(u, v)$ plane. To obtain an image of the average quiescent emission around an intrinsically variable $\mathrm{Sgr} \mathrm{A}^{*}$, it may therefore be necessary to average visibilities across multiple nights (R.-S. Lu et al., in preparation), although non-imaging techniques can still recover detailed spatial information on much shorter timescales (Doeleman et al. 2009b; Fish et al. 2009). The effects of refractive phase noise, small compared to those of source variability, will also be suppressed by this averaging process (Section 5.1).

\subsection{Applicability at $3.5 \mathrm{~mm}$}

The technique described in this paper will be applicable to Sgr A* at other wavelengths. For Sgr $\mathrm{A}^{*}$ at $1.3 \mathrm{~mm}$, the longest EHT baselines exceed the diffractive scale by less than a factor of two (Figure 1). At $3.5 \mathrm{~mm}$, most baselines among the inner seven telescopes of the Very Long Baseline Array (VLBA) are shorter than the diffractive scale. Baselines to the LMT and Green Bank Telescope (GBT) may nevertheless produce detectable fringes, since their large collecting area will offset additional losses from scattering on longer baselines. Scattering should not be so strong on baselines between the LMT and VLBA stations (with the exception of Mauna Kea) to prevent detection of Sgr A* (Figure 1). Baselines between phased ALMA and continental North America, though long, have a favorable orientation relative to the scattering ellipse. It is possible that Sgr A* may be detected on the very sensitive ALMA-LMT and ALMA-GBT baselines. However, the amount of correlated flux density on long baselines to ALMA (with projected baseline lengths ranging from $\sim 5000$ to $7200 \mathrm{~km}$ between ALMA and the VLBA sites of Fort Davis, Kitt Peak, Los Alamos, North Liberty, and Pie Town) will be strongly dependent on the intrinsic size of Sgr A* at $3.5 \mathrm{~mm}$ in these directions, estimated to be $\gtrsim 100 \mu$ as (Bower et al. 2006; Lu et al. 2011).

\section{CONCLUSIONS}

We have demonstrated that the scatter broadening of Sgr A* at $1.3 \mathrm{~mm}$ can be significantly mitigated. The predominant effect of interstellar scattering is to decrease the measured visibility amplitudes, especially on long baselines. As long as the observing array is sufficiently sensitive to obtain fringes on these baselines, visibility amplitudes can be corrected for this effect, recovering most of the unscattered structure of Sgr A*. Reconstructing the image from corrected visibilities produces a higher-fidelity image than attempting deconvolution after reconstructing an image from the uncorrected visibilities.

This result is of direct relevance for imaging $\mathrm{Sgr} \mathrm{A}^{*}$ with the EHT at $1.3 \mathrm{~mm}$ and may be applicable to longer wavelengths as well. It is also possible to construct non-imaging VLBI 
observables, such as closure phase and fractional polarization, that are unbiased by scatter broadening.

The Event Horizon Telescope is supported by grants from the National Science Foundation (NSF) and from the Gordon and Betty Moore Foundation (\#GBMF-3561). K.L.B. is partially supported by an NSF Graduate Fellowship. D.P. is supported by NASA/NSF TCAN award NNX14AB48G and by NSF award AST 1312034. R.N. was supported in part by NSF grant AST 1312651. A.E.B. receives financial support from the Perimeter Institute for Theoretical Physics and the Natural Sciences and Engineering Research Council of Canada through a Discovery Grant. Research at Perimeter Institute is supported by the Government of Canada through Industry Canada and by the Province of Ontario through the Ministry of Research and Innovation.

\section{REFERENCES}

Akiyama, K., Takahashi, R., Honma, M., Oyama, T., \& Kobayashi, H. 2013, PASJ, 65, 91

Armstrong, J. W., Rickett, B. J., \& Spangler, S. R. 1995, ApJ, 443, 209

Bardeen, J. M. 1973, in Black Holes (Les Astres Occlus), ed. C. DeWitt \& B. S. DeWitt (New York: Gordon and Breach), 215

Baron, F., Kloppenborg, B., \& Monnier, J. 2012, Proc. SPIE, 8445, 84451

Bower, G. C., Deller, A., Demorest, P., et al. 2014, ApJL, 780, L2

Bower, G. C., Falcke, H., Herrnstein, R. M., et al. 2004, Sci, 304, 704

Bower, G. C., Goss, W. M., Falcke, H., Backer, D. C., \& Lithwick, Y. 2006, ApJL, 648, L127

Broderick, A. E., Fish, V. L., Doeleman, S. S., \& Loeb, A. 2011a, ApJ, 735,110

Broderick, A. E., \& Loeb, A. 2005, MNRAS, 363, 353

Broderick, A. E., \& Loeb, A. 2006, MNRAS, 367, 905

Broderick, A. E., Loeb, A., \& Reid, M. J. 2011b, ApJ, 735, 57

Buscher, D. F. 1994, Very High Angular Resolution Imaging, 158, 91

Cornwell, T. J. 2008, ISTSP, 2, 793

Dexter, J., Agol, E., \& Fragile, P. C. 2009, ApJL, 703, L142

Dexter, J., Agol, E., Fragile, P. C., \& McKinney, J. C. 2010, ApJ, 717, 1092

Doeleman, S., Agol, E., Backer, D., et al. 2009a, Astro2010: The Astronomy and Astrophysics Decadal Survey, 2010, 68

Doeleman, S. S., Fish, V. L., Broderick, A. E., Loeb, A., \& Rogers, A. E. E. 2009b, ApJ, 695, 59

Doeleman, S. S., Shen, Z.-Q., Rogers, A. E. E., et al. 2001, AJ, 121, 2610

Doeleman, S. S., Weintroub, J., Rogers, A. E. E., et al. 2008, Natur, 455, 78

Eckart, A., Baganoff, F. K., Schdel, R., et al. 2006, A\&A, 450, 535

Falcke, H., Markoff, S., \& Bower, G. C. 2009, A\&A, 496, 77

Falcke, H., Melia, F., \& Agol, E. 2000, ApJL, 528, L13

Fish, V. L., Alef, W., Anderson, J., et al. 2013, High-AngularResolution and High-Sensitivity Science Enabled by Beamformed ALMA (arXiv:1309.3519)

Fish, V. L., Doeleman, S. S., Beaudoin, C., et al. 2011, ApJL, 727, L36
Fish, V. L., Doeleman, S. S., Broderick, A. E., Loeb, A., \& Rogers, A. E. E. 2009, ApJ, 706, 1353

Ghez, A. M., Salim, S., Weinberg, N. N., et al. 2008, ApJ, 689, 1044

Gillessen, S., Eisenhauer, F., Fritz, T. K., et al. 2009a, ApJL, 707, L114

Gillessen, S., Eisenhauer, F., Trippe, S., et al. 2009b, ApJ, 692, 1075

Goldreich, P., \& Sridhar, S. 1995, ApJ, 438, 763

Goodman, J., \& Narayan, R. 1989, MNRAS, 238, 995

Greisen, E. W., Spekkens, K., \& van Moorsel, G. A. 2009, AJ, 137, 4718

Gwinn, C. R., Britton, M. C., Reynolds, J. E., et al. 1998, ApJ, 505, 928

Gwinn, C. R., Danen, R. M., Middleditch, J., Ozernoy, L. M., \& Tran, T. Kh. 1991, ApJL, 381, L43

Gwinn, C. R., Kovalev, Y. Y., Johnson, M. D., \& Soglasnov, V. A. 2014, ApJL, 794, L14

Högbom, J. A. 1974, A\&AS, 15, 417

Jennison, R. C. 1958, MNRAS, 118, 276

Johannsen, T., \& Psaltis, D. 2010a, ApJ, 716, 187

Johannsen, T., \& Psaltis, D. 2010b, ApJ, 718, 446

Joshi, N., Zitnick, C. L., Szeliski, R., \& Kriegman, D. 2009, IEEE Conf. on Computer Vision and Pattern Recognition (Piscataway, NJ: IEEE), 1550

Krishnan, D., \& Fergus, R. 2009, in Proc. of Annual Conf. on Neural Information Processing Systems, Vol. 23, Advances in Neural Information Processing Systems 22, ed. Y. Bengio (Red Hook, NY: Curran \& Associates, Inc.), 1033

Lambert, H. C., \& Rickett, B. J. 1999, ApJ, 517, 299

Levin, A., Weiss, Y., Durand, F., \& Freeman, W. T. 2009, IEEE Conf. on Computer Vision and Pattern Recognition (Piscataway, NJ: IEEE), 1964

Levin, A., Weiss, Y., Durand, F., \& Freeman, W. T. 2011, IEEE Conf. on Computer Vision and Pattern Recognition (Piscataway, NJ: IEEE), 2657

Lo, K. Y., Shen, Q.-Z., Zhao, J.-H., \& Ho, P. T. P. 1998, ApJL, 508, L61

Łoza, A., Mihaylova, L., Bull, D., \& Canagarajah, N. 2009, Mach. Vis. Appl., 20,71

Lu, R.-S., Broderick, A. E., Baron, F., et al. 2014, ApJ, 788, 120

Lu, R.-S., Krichbaum, T. P., Eckart, A., et al. 2011, A\&A, 525, A76

Mościbrodzka, M., Gammie, C. F., Dolence, J. C., Shiokawa, H., \& Leung, P. K. 2009, ApJ, 706, 497

Narayan, R., \& Goodman, J. 1989, MNRAS, 238, 963

Noble, S. C., Leung, P. K., Gammie, C. F., \& Book, L. G. 2007, CQGra, 24, 259

Reid, M. J., Broderick, A. E., Loeb, A., Honma, M., \& Brunthaler, A. 2008, ApJ, 682, 1041

Rickett, B. J. 1990, ARA\&A, 28, 561

Rogers, A. E. E., Hinteregger, H. F., Whitney, A. R., et al. 1974, ApJ, 193, 293

Russ, J. C. 2011, The Image Processing Handbook (6th ed.; Boca Raton, FL; CRC Press)

Shen, Z.-Q., Lo, K. Y., Liang, M.-C., Ho, P. T. P., \& Zhao, J.-H. 2005, Natur, 438,62

Spangler, S. R., \& Gwinn, C. R. 1990, ApJL, 353, L29

Tatarskii, V. I. 1971, The Effects of the Turbulent Atmosphere on Wave Propagation (Jerusalem: Israel Program for Scientific Translations)

van Langevelde, H. J., Frail, D. A., Cordes, J. M., \& Diamond, P. J. 1992, ApJ, 396, 686

Wang, Z., Bovik, A. C., Sheikh, H. R., \& Simoncelli, E. P. 2004, ITIP, 13, 600

Wiener, N. 1949, The Extrapolation, Interpolation, and Smoothing of Stationary Time Series with Engineering Applications (Cambridge, MA: MIT Press)

Yan, W., Vincent, F. H., Abramowicz, M. A., Zdziarski, A. A., \& Straub, O. 2014, A\&A, submitted (arXiv:1406.0353)

Zoran, D., \& Weiss, Y. 2011, IEEE International Conf. on Computer Vision (Piscataway, NJ: IEEE), 479 